\title{
Open
}

\section{Epithelial-mesenchymal transformation and apoptosis in rat urethra development}

\author{
Yue Zhou ${ }^{1}$, Xing Liu ${ }^{1}$, Fangyuan Huang ${ }^{1}$, Yang Liu ${ }^{1}$, Xining Cao ${ }^{1}$, Lianju Shen ${ }^{1}$, Chunlan Long ${ }^{1}$, Dawei He ${ }^{1}$, Tao Lin ${ }^{1}$ and \\ Guanghui Wei ${ }^{1}$
}

BACKGROUND: To examine the mechanism of urethral seam formation during embryonal development of rat urethra.

METHODS: Time-mated Sprague-Dawley rats were killed and the genital tubercles of male pups harvested on embryonic day (ED) 15, 16, 18, and 19. External morphology was observed under scanning electron microscope. Serial transverse sections were prepared to examine dynamic changes in the urethral seam morphology with hematoxylin-eosin staining, immunohistochemistry, transmission electron microscopy, and double immunofluorescence.

RESULTS: Bilateral outgrowth of urethral swelling followed by urethral plate fusion in the midline to form urethral seam was observed from ED 16 onwards. Coexpression of epithelial and mesenchymal markers was observed in several cells at the urethral seam; a few cells with coexpression of epithelial and apoptotic markers were also observed. Mesenchymal transformation of epithelial cells and apoptotic epithelial cells was observed under transmission electron microscope.

CONCLUSION: Urethral formation occurs by tubulogenesis, which initiates proximally and progresses distally. This is the first study to demonstrate epithelial-mesenchymal transformation and epithelial cell apoptosis in the urethral seam cells of fetal rats. These findings provide new insights into the mechanisms involved in embryonal development of the urethra.

$\mathbf{H}$ ypospadias is the most common congenital malformation of the urogenital system, with an estimated incidence of one case per 200-300 male live births (1). An increasing trend in the number of reported cases of hypospadias has been observed over the years $(2,3)$. Several recent studies have sought to examine the causation of hypospadias from the environmental science, toxicological, and endocrinological perspectives (4-7). However, the etiopathogenesis of hypospadias remains largely unknown $(8,9)$. Understanding the normal urethral development can help identify the underlying mechanisms involved in the causation of hypospadias.
Embryology of the male urethra has been studied for decades; however, none of the proposed concepts explain the development of normal urethra in its entirety. According to the classical theory of fusion of urethral folds propounded by Glenister (10) and Hunter (11), urethral formation occurs due to the closure of bilateral urethral folds. However, the theory was rebuked by Kluth et al. (12) who found no evidence of fusion of urethral folds. Others have postulated an endodermal origin of the urethra, which later differentiates into the squamous epithelium $(13,14)$.

The urethral plate refers to the epithelial lining on the ventral part of the genital tubercle (GT), which is the key signal center in urethral formation (15). Urethral plate fusion generates the urethral seam $(16,17)$, and hence disorder of urethral seam fusion is a plausible mechanism that may lead to hypospadias $(18,19)$. Others have proposed that epithelialmesenchymal transformation (EMT), epithelial apoptosis, and epithelial cell migration are the key processes involved in the urethral seam formation. However, definitive evidence of the role of EMT or epithelial apoptosis is yet to be obtained (20).

The aim of our study was to elucidate the morphology and basic processes involved in the embryonic development of the male rat urethra, with a special focus on the role of EMT and apoptosis in urethral seam fusion.

\section{METHODS}

\section{Animals}

Twenty time-mated Sprague-Dawley rats were used in this study. These were obtained from the Experimental Animal Center of the Chongqing Medical University (Chongqing, China; SPF, License No.: SYXK (Chongqing) 2007-0001) and were raised at the Experimental Animal Center at the Children's Hospital of Chongqing Medical University (SPF, License No.: SYXK (Chongqing) 2007-0016). All experiments involving animals were approved by the Ethics Committee of Chongqing Medical University (license number: SCXK[YU]20110016). The morning on which the vaginal plug was found was considered as the embryonic day (ED) 0 . Gender of the embryos was confirmed using polymerase chain reaction for the SRY gene. On EDs 15, 16, 18, and 19, the GT of male embryos was harvested.

\footnotetext{
'Department of Pediatric Urology Surgery, Children's Hospital of Chongqing Medical University, Ministry of Education Key Laboratory of Child Development and Disorders, China International Science and Technology Cooperation Base of Child Development and Critical Disorders, Chongqing Key Laboratory of Pediatrics, Chongqing, China. Correspondence: Xing Liu (liux_0217@163.com) 


\section{Articles | zhou et al.}

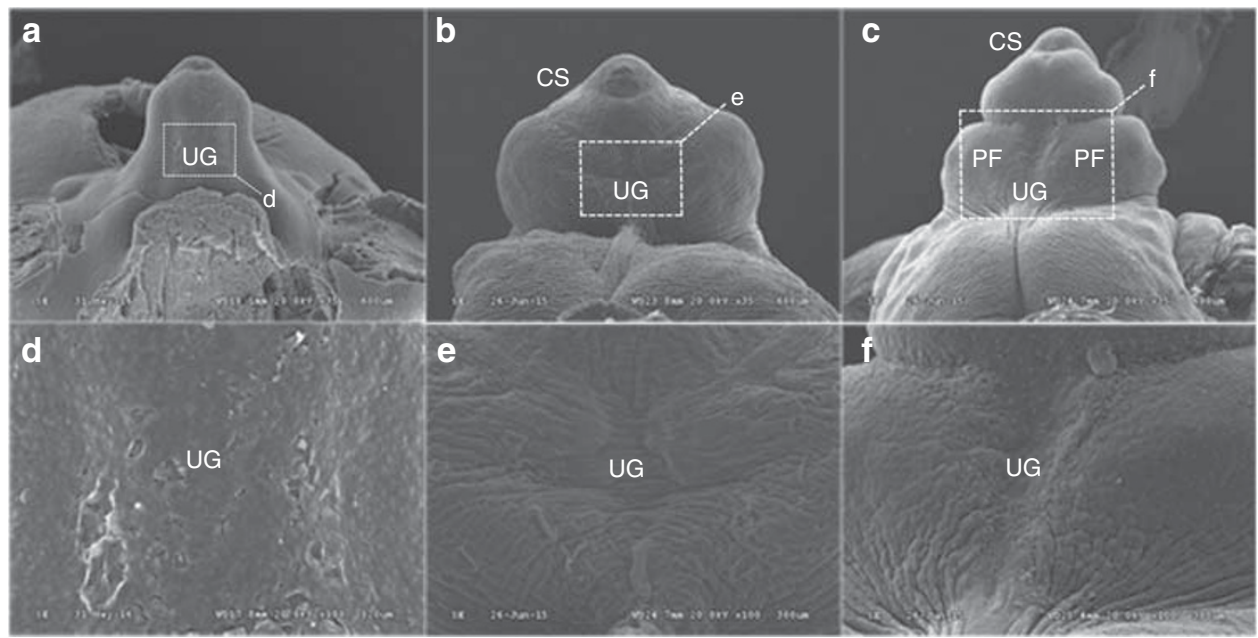

Figure 1. Gross images of genital tubercle at different embryonic stages under SEM. Ventral view of embryonic genital tubercle on ED 15 (a), ED 16 (b), and ED 18 (c) under SEM. Tail and lower limbs were removed. With progression of embryonic time, elongation of the genital tubercle, deepening of the urethral groove, and emergence of coronary sulcus can be seen. Magnified views of the urethral groove area (marked with a dotted line rectangle in the upper panels) are presented in the respective lower panels (d-f). Urethral groove appears as a complete structure with no signs of a canal inside it. CS, coronary sulcus; ED, embryonic day; PF, preputial fold; SEM, scanning electron microscope; UG, urethral groove.

\section{Histological and Immunohistochemical Examination}

Penis samples obtained at different time points were formalin-fixed, dehydrated, and paraffin-embedded. Serial transverse sections $(4 \mu \mathrm{m})$ stained with hematoxylin and eosin were observed under electron microscope (Nikon, 930195, Tokyo, Japan). After antigen retrieval and blockage with bovine serum albumin (ZLI-9027, ZSGB, China), sections of samples obtained after ED 15 were incubated with antibodies against pan-cytokeratin (CK-pan, ab7753, Abcam, UK, $1: 3,000$, used to label epithelial cells) to highlight the outside skin and inner urethra.

\section{Scanning Electron Microscopy}

The penis specimens were washed in normal saline, fixed with glutaraldehyde, dehydrated, and gold-coated for observation under scanning electron microscope (SEM; Hitachi, S-3000N, Tokyo, Japan).

\section{Transmission Electron Microscope}

Penis specimens were collected at ED 19 and fixed in glutaraldehyde. After fixation, the samples were dehydrated with incremental concentrations of ethanol, embedded in pure resin, and $20 \mu \mathrm{m}$ sections prepared with ultramicrotome. These sections were examined under a transmission electron microscope (Hitachi, S-3000N) and photographs obtained.

\section{Double Immunofluorescence}

For double immunofluorescent staining, paraffin sections were incubated with primary antibodies against E-cadherin (CST, \#14472, Boston, MA, 1:50, used to label epithelial cells) and $\alpha$ smooth muscle actin (Abcam, ab124964, 1:300, used to label cells of mesenchymal origin), or ZO-1 (Invitrogen, \#61-7300, Carlsbad, CA, $1: 50$, used to label epithelial cells) and N-cadherin (Invitrogen, \#33$3900,1: 100$, used to label cells of mesenchymal origin), or of CK-pan (Abcam, ab7753, UK, 1:3,000, used to label epithelial cells) and cleaved-caspase 3 (caspase3, CST, \#9664, 1:350, used to label apoptotic cells) at $4{ }^{\circ} \mathrm{C}$ overnight after de-paraffinization, hydration, and blockage in $0.5 \%$ bovine serum albumin. On the following day, the sections were incubated with corresponding secondary antibodies for $1 \mathrm{~h}$ at room temperature. After nuclear counterstaining with diamidino-phenyl-indole, images were examined under a fluorescence microscope (Nikon, K10587).

\section{RESULTS}

\section{Urethral Morphogenesis}

To understand the development of rat GTs dynamically, we documented the overview of GTs from ED 15 to ED 19. On ED 15, external genitalia of male embryonic rats appeared as a short conical structure (Figure 1a), and a faint urethral groove was seen at the front ventral part of GT (Figure 1d). On ED 16, external genitalia were found to have assumed a cylindrical shape. At this time, coronary sulcus appeared at the tip of GT (Figure 1b). At the ventral part, the urethral groove deepened at a point proximal to the coronary sulcus and not at the tip of the urethra. Preputial folds started appearing around the penis (Figure 1e). On ED 18, external genitalia were longer than before, and a midline groove was observed in the external genitalia (Figure 1f). Besides, glans penis and the penile shaft were clearly differentiated and were divided by the coronary sulcus. The skin folds were obvious on both sides of the groove (Figure 1c). The general view on ED 19 was similar to that on ED 18, and hence the corresponding image has not been included in the figure below.

\section{Urethral Seam Formation}

To further study the formation of the urethra, serial sections were prepared from the tip to the bottom of GT to examine the internal structure. From distal to proximal, increase in the transversal area was observed. In the apical section, the midventral epithelium or the urethral plate formed a bend into the mesenchyme with growth of bilateral urethral swellings (Figure $\mathbf{2 b}-\mathbf{d}$ ). Closer to the proximal urethra, the bend deepened into a hollow central canal (Figure 2e,f). Simultaneously, the two-layered epithelium grew progressively, approximated each other (Figure $2 \mathrm{~g}$ ), and finally fused 


\section{EMT and apoptosis in the urethra Articles}

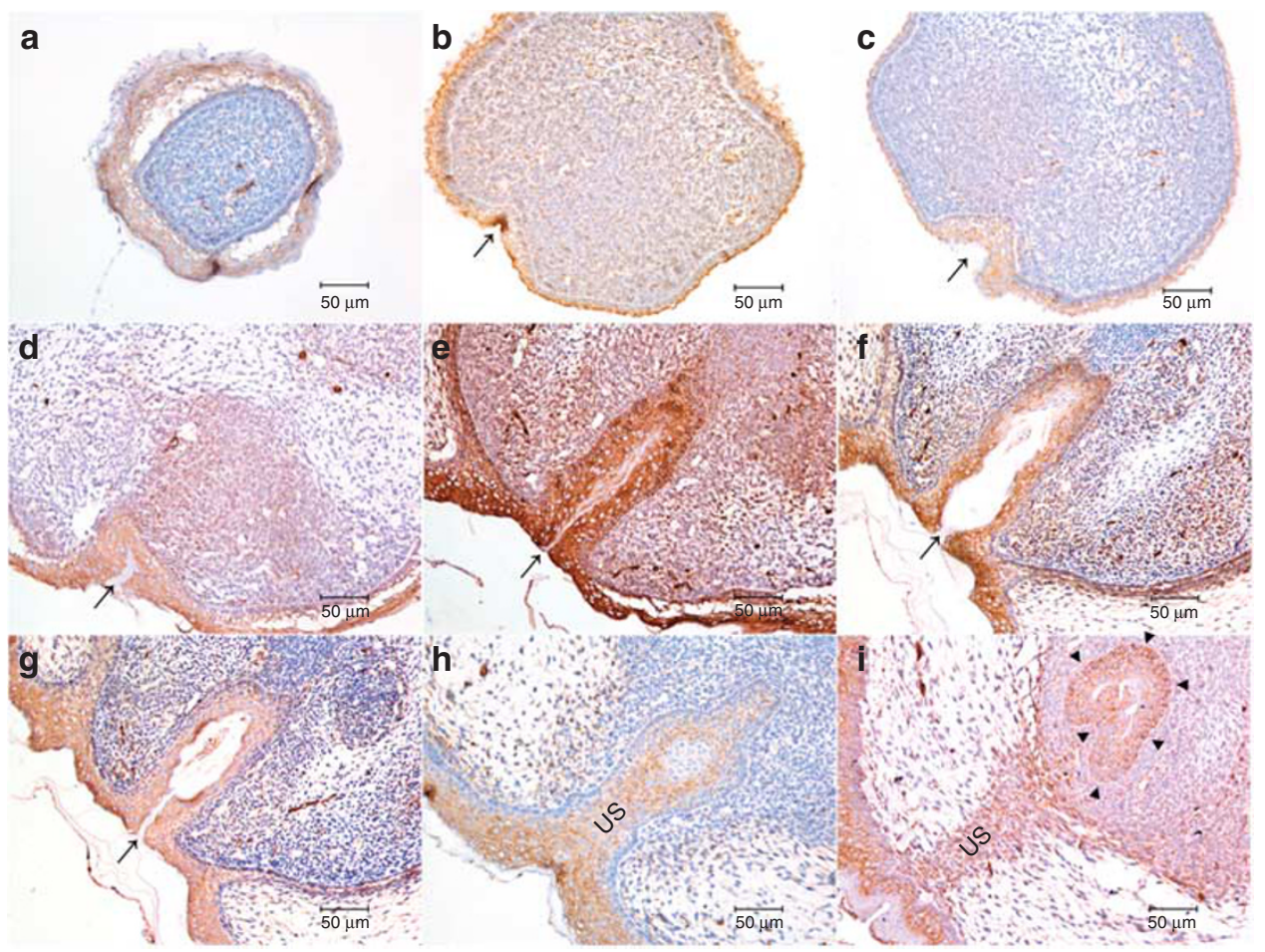

Figure 2. Immunohistochemical staining of rat penis sections with CK-pan at ED 19. (a-i) Representation of sections from distal to proximal ( $\times 200)$. (a) Transverse section of the tip of the penis is oval with a layer of epithelium surrounding the mesenchyme. (b) A shallow invagination (arrow, indicating the urethral plate) has appeared at the ventral penis. (c) The invagination of bilateral urethral plate has deepened (arrow). (d) " $U$ "-shaped fold (arrow) is formed. (e) Urethral plate has grown and the fold has invaginated into the mesenchyme. (f) With elongation of the urethral plate, "O"shaped urethra cavity is formed, but is not intact. (g) The two layered-urethral plates have drawn nearer, but do not meet. (h) Urethral plate is fused and the urethral seam can be seen; the center of urethral plate is the primitive urethra. (i) Urethral seam has remodeled and forms the inner mature urethra (arrowhead). CK-pan, pan-cytokeratin; ED, embryonic day; US, urethral seam.

in the middle line to form a urethral seam (Figure $2 \mathbf{h}$ ). Nearer to the basal urethra, the epithelial cells of urethral seam disappeared, remodeled into the inner urethral epithelium and outer skin, and the urethra formed (Figure 2i). Urethral plate development of embryonic rats on ED 15, 16, 18, and 19 underwent the similar process. From the overview of GTs, we observed that prepuce appeared around the penis after ED 15. CK-pan was used to outline the preputial space. All these processes showed that urethra formation occurred by tubulogenesis (Figure 2). During the process of GT development, a membrane was found covering the primitive urethra, under the ventral bottom of the GT (Figure 3).

\section{Epithelial-Mesenchymal Transformation}

The above observations showed that the urethral seam was formed via urethral plate fusion, and the urethral seam in the lower part of penis was totally formed, began to remodel (Figure $4 \mathrm{~b}$ ). The question now was what happened to those epithelial cells in the urethral seam during fusion? In order to investigate whether the epithelial cells of urethral plate transformed into mesenchymal cells, we examined these cells with double immunofluorescence. Unexpectedly, some cells expressed E-cadherin while also expressing $\alpha$-smooth muscle actin (Figure 4c). Similarly, concomitant expression of epithelial and mesenchymal markers, ZO-1 and N-cadherin, was also observed (Figure 4d). Subsequently, the phenomenon of EMT was tested further by transmission electron microscope. Many cells with large nuclei and scant cytoplasm were observed scattered in the urethral seam. Among these, several cells were found closely arranged, which had lost their intercellular junctions, and showed only some desmosomal remanants; the formerly smooth basal surface exhibited a filopodial appearance (Figure 5). Epithelial cells underwent profound changes in cell shape such as disappearance of the midline epithelial seam, gradual loss of epithelial characteristics, and acquisition of mesenchymal characteristics, which indicate that EMT did occur during the development of the urethra.

\section{Epithelial Cell Apoptosis}

Immunofluorescent co-staining with antibodies against CKpan and caspase 3 was performed to assess whether epithelial apoptosis was involved in urethral seam formation. Along with the expression of CK-pan, a few cells that stained positive for the apoptotic marker, cleave-caspase 3 , and dense clumps of nuclear material were observed (Figure 6a). Under transmission electron microscope, several cells exhibited cell membrane shrinkage, chromatin margination, degeneration of cells surrounding the cell junctions, and disappearance of 


\section{Articles | zhou et al.}

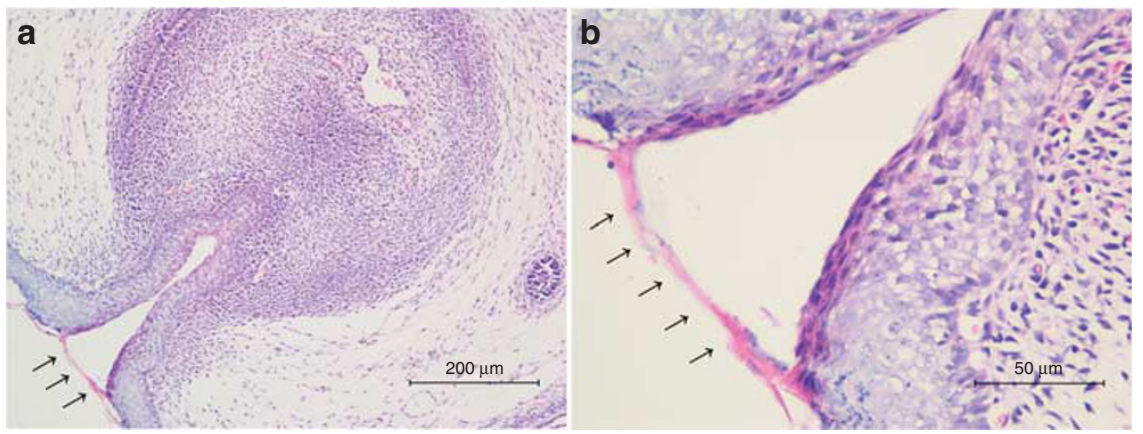

Figure 3. HE-stained section of rat genital tubercle on ED 19. (a) At the ventral aspect of the GT, a U-shaped invagination of the urethral plate into mesenchyme is seen. A membrane is seen bridging the recess caused by the invagination (arrows; $\times 200)$. (b) Magnified view shows that the thin membrane is connected with the edge of the urethral plate, and thus forms a closed urethral cavity ( $\times 400)$. ED, embryonic day; GT, genital tubercle; $\mathrm{HE}$, hematoxylin and eosin.

a

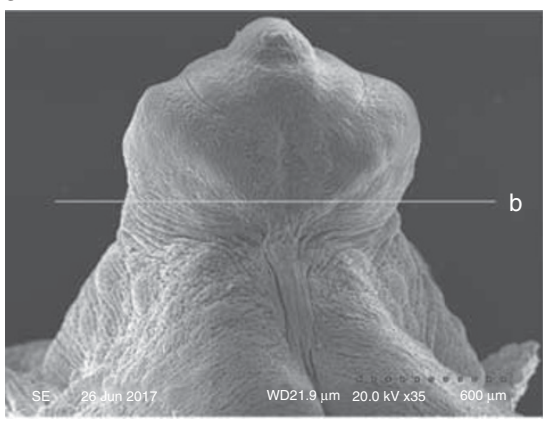

C

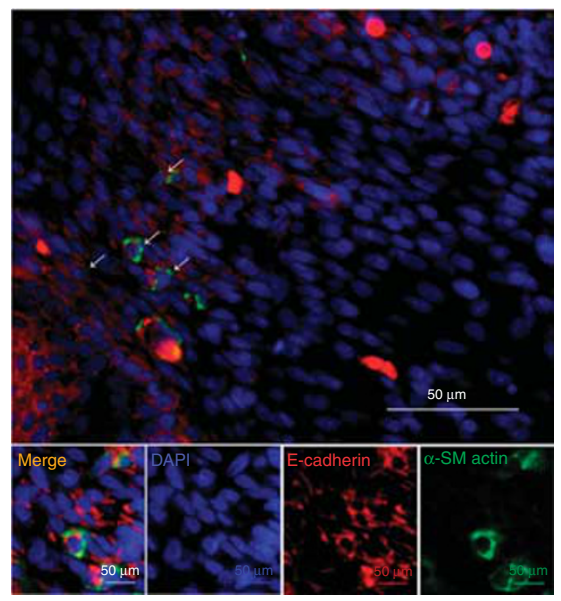

b

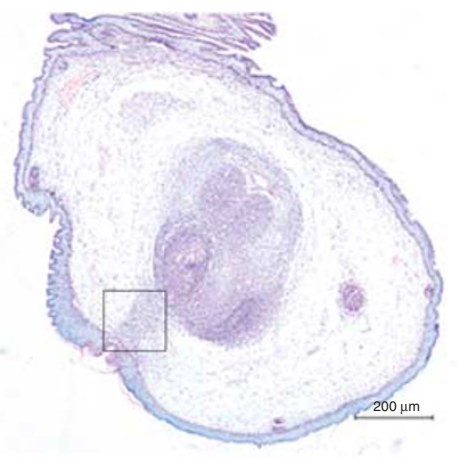

d

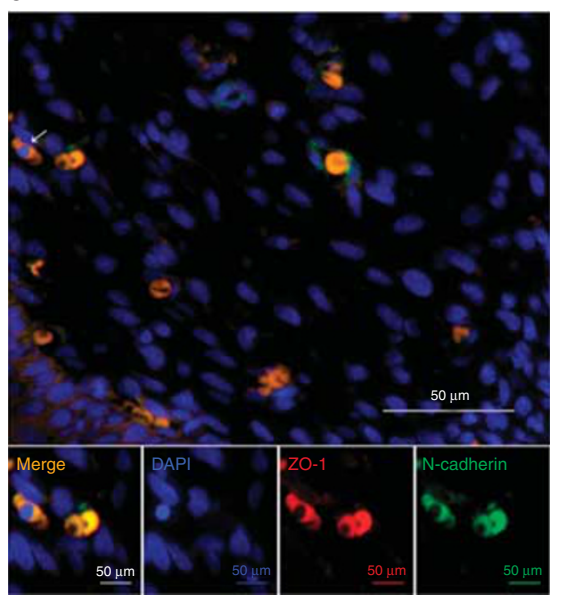

Figure 4. Demonstration of epithelial-mesenchymal transition (EMT) by tracing urethral seam cells with double immunofluorescent staining. (a) Scanning electron microscopy of an embryonic penis at ED 19 from ventral view; transverse section at lower part of the embryonic penis is seen in (b). (b) HE-stained section of urethral seam at ED $19(\times 40)$. Urethral plate has fused totally and urethral seam is remodeling. The inset, which stands for urethral seam, is magnified in $(\mathbf{c}, \mathbf{d})$. (c,d) Immunofluorescent staining of the urethral seam for EMT on ED 19 ( $\times 400)$. Double immunofluorescence for epithelial marker E-cadherin and mesenchymal marker a-SM actin (c) and epithelial marker ZO-1 and mesenchymal marker N-cadherin (d). When merged together, some cells coexpress epithelial and mesenchymal markers (arrow); nuclei are counterstained by DAPI. a-SM, a-smooth muscle actin; $\mathrm{CS}$, coronary sulcus; DAPI, diamidino-phenyl-indole; ED, embryonic day; EMT, epithelial-mesenchymal transformation; $\mathrm{HE}$, hematoxylin and eosin; SC, scrotum; UO, urethral opening; UP, urethral plate.

tight junctions (Figure 6b). These findings were unlike those of normal epithelial cells that exhibit a uniform tight arrangement and interact with each other via cell junctions. The findings illustrate that epithelial apoptosis was involved in urethral seam formation.

\section{DISCUSSION}

The embryology of urethral development has been a subject of debate since many years. The proposed mechanisms such as tubulogenesis of GT $(21,22)$, ectodermal ingrowth theory $(23,24)$, and endodermal differentiation theory $(17,25)$ do not 


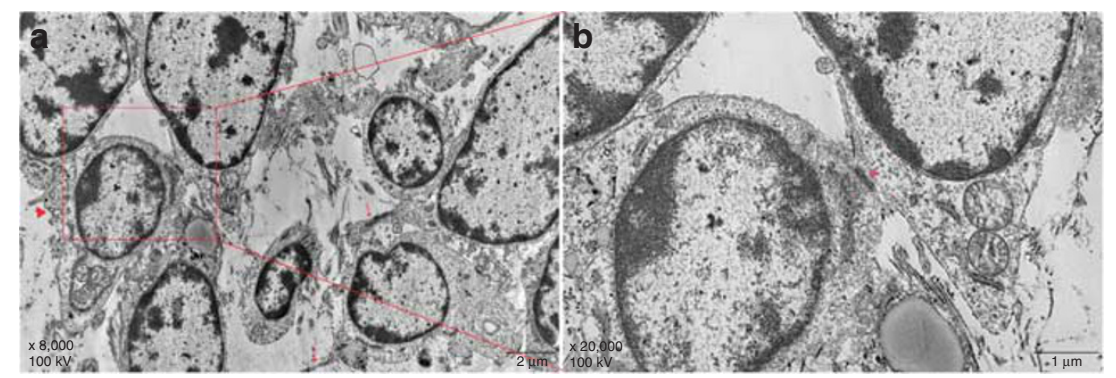

Figure 5. Demonstration of EMT by tracing urethral seam cells with transmission electron microscope. (a) Mesenchymal cells were distinguished morphologically by their shape and by well-developed pseudopodia and filopodia (arrow). The epithelial island is the remnant of the disappearing urethral seam. The epithelial cells in the island were joined by desmosomes, one of which (square inset) is enlarged in (b) (asterisk). Note the appearance of filopodia (arrowhead) of the epithelial cells. (b) Inset in (a) shows a magnified view of desmosome (asterisk).
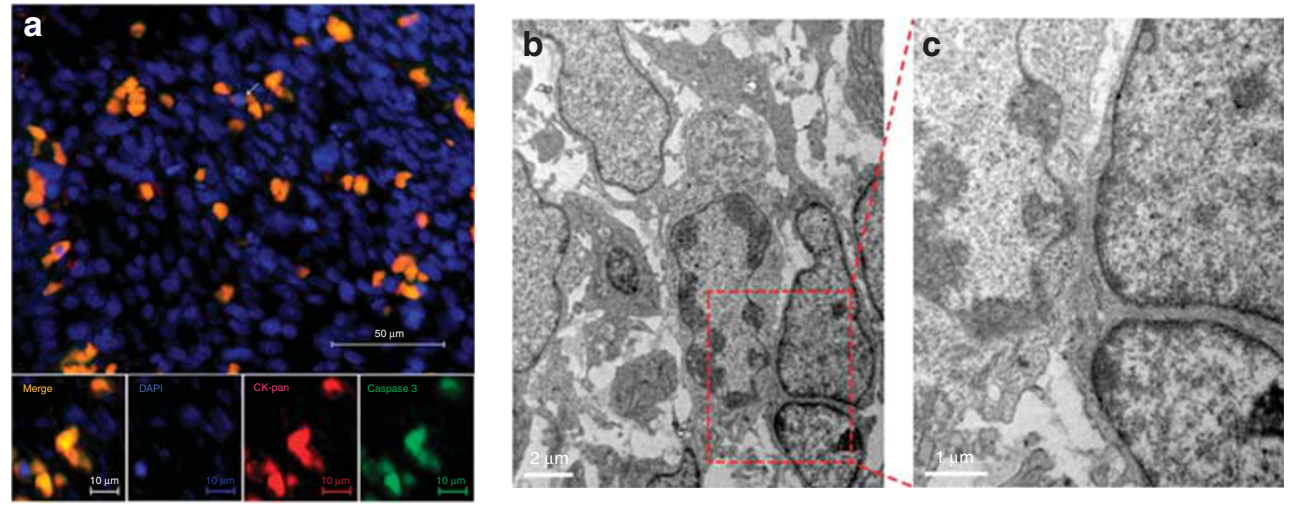

Figure 6. Representation of epithelial apoptosis in urethral seam cells. (a) Immunofluorescent staining of urethral seam at ED 19 with CK-pan (red) and cleaved-caspase 3 (green; $\times 400$ ). Numerous cells are seen scattered along the urethral seam, visualized with DAPI. Among these, punctate distribution of cells with deeply stained nuclear matter and coexpression of CK-pan and cleaved-caspase 3 is seen (indicated by arrow). (b) Ultrastructure of cells at urethral seam at ED 19 observed under transmission electron microscopy. Among the matrix of urethral seam, shrinkage of cell membrane and margination of chromatin is observed. (c) Magnified view of intercell junctions (dotted box in b) showing degeneration and even disappearance of some tight junctions. CK-pan, pan-cytokeratin; DAPI, diamidino-phenyl-indole; ED, embryonic day.

explain the whole disease spectrum. Tissue fusion is an important mechanism in embryonal development and organ formation, such as in the heart, neural tube, and palate (26-28). Disruption of these processes underlies several congenital disorders such heart defects, spina bifida, and cleft palate. In this study, by way of serial sections, we observed outgrowth of bilateral urethral folds and apposition and fusion of the urethral plate from a proximal to distal direction, which formed a zipper shape. These findings indicate that urethral development was through tubulogenesis, as verified by other researchers $(11,21,29)$.

Presence of the cloacal membrane at the bottom of the urogenital sinus has been shown in rabbits, pigs, and human embryos (29-34). Kluth et al. (35) also documented the presence of cloacal membrane at the ventral surface of the urethra in rats under SEM; however, they did not observe the fusion of urethral folds. On this premise, they concluded that the urethra derives entirely from the endoderm of the primitive hindgut. Besides SEM, we examined serial sections to characterize in detail the development of the urethral inner structures. As depicted in Figures 2 and 3, the urethral folds continued to grow toward the midline under the cloacal membrane, and the urethral plate invaginated and fused to form the urethral seam. Therefore, we believe that urethral development was through tubulogenesis.

During fusion of urethral folds, the fate of epithelial cells in the urethral seam has been widely contested for years. Similar to that observed during embryonic palate development, EMT, apoptosis, and epithelial cell migration were hypothesized to be involved in urethral seam formation $(18,20)$. The pivotal role of EMT in embryogenesis, including in neural crest development, secondary palate formation, heart valve development, and male Mullerian duct regression, is well documented (36,37); EMT dysregulation at the embryonic stage can induce congenital malformations. Some researchers proposed that cellular migration rather than EMT or epithelial apoptosis is involved in urethral seam formation (20). They attributed their claim to the lack of mesenchymal phenotypes in the epithelial cells in the urethral seam; they instead proposed a change in the orientation and polarization 


\section{Articles | Zhou et al.}

of epithelial cells. Thus, they concluded that urethral seam remodeling was through cell migration and not EMT. However, in our study, we found coexpression of epithelial and mesenchymal markers in the urethral seam (Figure 4), degeneration of intercellular junctions, and formation of filopodia (Figure 5), which indicates that EMT is indeed involved in embryonal penile development. We presume that the distinct outcomes were the result of choice of the experimental animal. The width of embryonic urethral seam $(\sim 30 \mu \mathrm{m})$ in mouse is much narrower than that in rats, which allows a relatively large volume of epithelial cells to transgress into the surrounding matrix and migrate to the outer epithelium or the inner urothelium. Therefore, we opted for the rat model as their relatively wide seam facilitates closer examination of urethral seam remodeling.

Apoptosis is considered essential in normal embryonal development $(38,39)$, for instance, for the regression of anuran's tail. Hadidi et al. (21) did not report any conclusive evidence of epithelial apoptosis. In the study by Baskin et al. (20), apoptosis occurred among mesenchymal cells in the urethral seam of mouse. However, they found no evidence of epithelial cell apoptosis. Our findings represent a definitive evidence of epithelial apoptosis, as depicted in Figure 5. We believe that the difference is attributable to the different animal models used. In the narrow seam of mouse, most epithelial cells migrated to the nearby epithelium; the rest of the cells that were too few to be caught even underwent transient apoptosis. In rats, a wide urethral seam and more epithelial cells were allowed for observation. Therefore, rat can be an ideal animal model for such experiments.

With the help of double fluorescence, we identified the following mechanisms involved in normal development of the rat urethra: (i) tubulogenesis; (ii) urethral plate fusion; (iii) EMT; and (iv) epithelial cell apoptosis. Our findings are of much relevance and can help unravel the mechanisms involved in congenital malformations such as hypospadias.

A limitation of our work was that we did not observe any orifices clearly under the SEM; this may be attributable to the peculiar orientation of embryonic species. However, we compensated for this by examination of serial sections up to the actual fusion point. Even though there were several cells found undergoing EMT or apoptosis on a certain section, we believe that overall, only some cells were undergoing this process and most cells stayed relatively static. The most appropriate approach would be to investigate these findings in human embryos in a future study.

\section{CONCLUSION}

Rat urethral development involves tubulogenesis at the GT and urethral plate fusion to form the urethral seam. EMT and epithelial cell apoptosis have a role in urethral seam fusion. Aberration of any of these processes during embryonal development may potentially be involved in the pathogenesis of hypospadias.

\section{STATEMENT OF FINANCIAL SUPPORT}

This study was supported by the National Natural Science Foundation of China (grant 81370782) and the National Natural Science Foundation of China (grant 81370701).

Disclosure: The authors declare no conflict of interest.

\section{REFERENCES}

1. Bouty A, Ayers KL, Pask A, Heloury Y, Sinclair AH. The genetic and environmental factors underlying hypospadias. Sex Dev 2015;9:239-59.

2. Canon S, Mosley B, Chipollini J, Purifoy JA, Hobbs C. Epidemiological assessment of hypospadias by degree of severity. J Urol 2012;188:2362-6.

3. Nordenvall AS, Frisén L, Nordenström A, Lichtenstein P, Nordenskjöld A. Population based nationwide study of hypospadias in Sweden, 1973 to 2009: incidence and risk factors. J Urol 2014;191:783-9.

4. Carmichael SL, Shaw GM, Lammer EJ. Environmental and genetic contributors to hypospadias: a review of the epidemiologic evidence. Birth Defects Res A Clin Mol Teratol 2012;94:499-510.

5. Winston JJ, Meyer RE, Emch ME. Geographic analysis of individual and environmental risk factors for hypospadias births. Birth Defects Res A Clin Mol Teratol 2014;100:887-94.

6. Leung MC, Phuong J, Baker NC, et al. Systems toxicology of male reproductive development: profiling 774 chemicals for molecular targets and adverse outcomes. Environ Health Perspect 2016;124:1050-61.

7. Michalakis M, Tzatzarakis MN, Kovatsi L, et al. Hypospadias in offspring is associated with chronic exposure of parents to organophosphate and organochlorine pesticides. Toxicol Lett 2014;230:139-45.

8. van der Zanden LF, van Rooij IA, Feitz WF, Franke B, Knoers NV, Roeleveld N. Aetiology of hypospadias: a systematic review of genes and environment. Hum Reprod Update 2012;18:260-83.

9. Baskin LS, Ebbers MB. Hypospadias: anatomy, etiology, and technique. J Pediatr Surg 2006;41:463-72.

10. Glenister TW. The origin and fate of the urethral plate in man. J Anat 1954;88:413-25.

11. Hunter RH. Notes on the development of the prepuce. J Anat 1935;70: 68-75.

12. Kluth D, Lambrecht W, Reich P. Pathogenesis of hypospadias-more questions than answers. J Pediatr Surg 1988;23:1095-101.

13. Hynes PJ, Fraher JP. The development of the male genitourinary system: III. The formation of the spongiose and glandar urethra. Br J Plast Surg 2004;57:203-14.

14. Wood-Jones F. Report LXXXIX: the nature of the malformations of the rectum and urogenital passages. Br Med J 1904;2:1630-4.

15. Haraguchi R, Mo R, Hui C, et al. Unique functions of Sonic hedgehog signaling during external genitalia development. Development 2001;128: 4241-50.

16. Baskin LS, Lee YT, Cunha GR. Neuroanatomical ontogeny of the human fetal penis. Br J Urol 1997;79:628-40.

17. Kurzrock EA, Baskin LS, Cunha GR. Ontogeny of the male urethra: theory of endodermal differentiation. Differentiation 1999;64:115-22.

18. Seifert AW, Harfe BD, Cohn MJ. Cell lineage analysis demonstrates an endodermal origin of the distal urethra and perineum. Dev Biol 2008;318: 143-52.

19. Yamada G, Satoh Y, Baskin LS, Cunha GR. Cellular and molecular mechanisms of development of the external genitalia. Differentiation 2003;71:445-60.

20. Baskin LS, Erol A, Jegatheesan P, Li Y, Liu W, Cunha GR. Urethral seam formation and hypospadias. Cell Tissue Res 2001;305:379-87.

21. Hadidi AT, Roessler J, Coerdt W. Development of the human male urethra: a histochemical study on human embryos. J Pediatr Surg 2014;49:1146-52.

22. Gredler ML, Seifert AW, Cohn MJ. Tissue-specific roles of Fgfr2 in development of the external genitalia. Development 2015;142:2203-12.

23. Hart DB. On the rôle of the developing epidermis in forming sheaths and lumina to organs, illustrated specially in the development of the prepuce and urethra. J Anat Physiol 1907;42:50-6.

24. Jones FW. The development and malformations of the glans and prepuce. Br Med J 1910;1:137-8. 
25. Baskin LS. Hypospadias and urethral development. J Urol 2000;163: 951-6.

26. Ray HJ, Niswander L. Mechanisms of tissue fusion during development. Development 2012;139:1701-11.

27. Holtgrave EA, Stoltenburg-Didinger G. Apoptotic epithelial cell death: a prerequisite for palatal fusion. An in vivo study in rabbits. J Craniomaxillofac Surg 2002;30:329-36.

28. Nievelstein RA, Hartwig NG, Vermeij-Keers C, Valk J. Embryonic development of the mammalian caudal neural tube. Teratology 1993;48:21-31.

29. van der Werff JF, Nievelstein RA, Brands E, Luijsterburg AJ, VermeijKeers C. Normal development of the male anterior urethra. Teratology 2000;61:172-83.

30. Miyagawa S, Harada M, Matsumaru D, et al. Disruption of the temporally regulated cloaca endodermal $\beta$-catenin signaling causes anorectal malformations. Cell Death Differ 2014;21:990-7.

31. Ching ST, Cunha GR, Baskin LS, Basson MA, Klein OD. Coordinated activity of Spry1 and Spry2 is required for normal development of the external genitalia. Dev Biol 2014;386:1-11.

32. Müller F, O'Rahilly R. The primitive streak, the caudal eminence and related structures in staged human embryos. Cells Tissues Organs 2004;177:2-20.

33. Matsumaru D, Murashima A, Fukushima J, et al. Systematic stereoscopic analyses for cloacal development: the origin of anorectal malformations. Sci Rep 2015;5:13943.

34. Hassoun R, Schwartz P, Rath D, Viebahn C, Männer J. Germ layer differentiation during early hindgut and cloaca formation in rabbit and pig embryos. J Anat 2010;217:665-78.
35. Kluth D, Fiegel HC, Geyer C, Metzger R. Embryology of the distal urethra and external genitals. Semin Pediatr Surg 2011;20:176-87.

36. Yang J, Weinberg RA. Epithelial-mesenchymal transition: at the crossroads of development and tumor metastasis. Dev Cell 2008;14:818-29.

37. Moustakas A, Heldin CH. Signaling networks guiding epithelialmesenchymal transitions during embryogenesis and cancer progression. Cancer Sci 2007;98:1512-20.

38. Clarke PG, Clarke S. Nineteenth century research on naturally occurring cell death and related phenomena. Anat Embryol 1996;193:81-99.

39. Glucksmann A. Cell deaths in normal vertebrate ontogeny. Biol Rev Camb Philos Soc 1951;26:59-86.

(c) (1) (8) $\Theta$ This work is licensed under a Creative Commons Attribution-NonCommercial-NoDerivs

International License. The images or other third party material in this article are included in the article's Creative Commons license, unless indicated otherwise in the credit line; if the material is not included under the Creative Commons license, users will need to obtain permission from the license holder to reproduce the material. To view a copy of this license, visit http://creativecommons.org/licenses/ by-nc-nd/4.0/

(c) The Author(s) (2017) 